\title{
Is There an Orbital Signal in the Polar Layered Deposits on Mars?
}

\section{Citation}

Perron, J. Taylor, and Peter John Huybers. 2009. Is there an orbital signal in the polar layered deposits on Mars? Geology 37(2): 155-158.

\section{Published Version}

http://dx.doi.org/10.1130/G25143A.1

\section{Permanent link}

http://nrs.harvard.edu/urn-3:HUL.InstRepos:3356132

\section{Terms of Use}

This article was downloaded from Harvard University's DASH repository, and is made available under the terms and conditions applicable to Other Posted Material, as set forth at http:// nrs.harvard.edu/urn-3:HUL.InstRepos:dash.current.terms-of-use\#LAA

\section{Share Your Story}

The Harvard community has made this article openly available.

Please share how this access benefits you. Submit a story.

Accessibility 


\title{
Is there an orbital signal in the polar layered deposits on Mars?
}

\author{
J. Taylor Perron*, Peter Huybers \\ Department of Earth and Planetary Sciences, Harvard University, Cambridge, Massachusetts 02138, USA
}

\begin{abstract}
Do the polar layered deposits on Mars reflect orbital control or stochastic variability? It is first useful to determine whether an orbital signal would be detected, even if present. An estimate of the uncertainty in the time-depth relationship of the polar stratigraphy shows that nonlinearities in this relationship and noise in the signal will hamper or preclude detection of orbital forcing, even if layer composition is directly proportional to insolation. Indeed, stratigraphic sections of the north polar layered deposits reconstructed from spacecraft images yield no clear evidence of orbital control and are largely consistent with an autoregressive, stochastic formation process. There is, however, a broad rise in spectral power centered on a wavelength of roughly $1.6 \mathrm{~m}$ that appears in many of the stratigraphic sections. This bedding may record a time scale associated with processes internal to Mars' climate system, perhaps related to dust storms. Alternatively, if formed in response to variations in Mars' obliquity or orbital precession, the $1.6 \mathrm{~m}$ bedding implies that the $\sim 1-\mathrm{km}$-thick upper north polar layered deposits formed over 30-70 Myr.
\end{abstract}

\section{INTRODUCTION}

Polar layered deposits (PLD)—stratigraphic sequences of ice and dust that have accumulated to thicknesses of a few kilometers at both poles-provide the most promising record of recent climate variability on Mars, but the origin of the variability and the duration of the record remain unknown. Since the Mariner and Viking orbiters observed alternating light and dark layers exposed near the margins of Mars' polar caps, planetary scientists have speculated that the PLD contain a record of ice and dust accumulation controlled by the insolation variations caused by changes in Mars' orbital configuration, particularly the 120 kyr obliquity and 51 kyr precession periods (e.g., Murray et al., 1972; Cutts et al., 1976; Toon et al., 1980; Cutts and Lewis, 1982; Howard et al., 1982; Thomas et al., 1992). Highresolution images and radar soundings from subsequent missions have revealed a more complex stratigraphy, which has also been hypothesized to contain orbital features (Laskar et al., 2002; Milkovich and Head, 2005; Fishbaugh et al., 2008; Phillips et al., 2008).

A similar case is often made for paleoclimate records on Earth (e.g., Hays et al., 1976), but it is worth noting that no more than $20 \%$ of the variability in late Pleistocene climate (as recorded in marine $\delta^{18} \mathrm{O}$ and $\delta \mathrm{D}$ ) is linearly attributable to variations in Earth's orbital configuration (Wunsch, 2004). A long-running debate within the terrestrial paleoclimate community concerns the extent to which Earth's glacial variability is deterministically controlled by orbital variations (e.g., Kominz and Pisias, 1979; Wunsch, 2004).

Mars is subject to larger orbital variations in insolation than Earth (Ward, 1973; Laskar

*Current address: Department of Earth, Atmospheric and Planetary Sciences, Massachusetts Institute of Technology, Cambridge, Massachusetts 02139; E-mail: perron@mit.edu. et al., 2004), has a thin atmosphere and no oceans, and thus may appear to have a simpler climate system, but the potential for nonlinear climate responses is significant, particularly in the dynamics of large dust storms (Pollack et al., 1979; Toon et al., 1980; Haberle, 1986; Zurek and Martin, 1993). For Mars, as for Earth, we must weigh the null hypothesis that the record of climate variability is either unrelated to shifts in orbital configuration or is related in a sufficiently complicated way that identification of the orbital imprint is not possible.

\section{DETECTABILITY OF A NOISY ORBITAL SIGNAL}

To evaluate the feasibility of recovering an orbital signal, we construct a model of the north PLD (NPLD) stratigraphy from spacecraft images, measure the variability in vertical distance between layers, and examine the effect of this stratigraphic variability on the spectral representation of a simple orbital signal. We select six Mars Orbiter Camera (MOC) images within a $10,000 \mathrm{~km}^{2}$ region in which the same stratigraphic sequence is exposed along gently sloping troughs eroded into the surface of the north polar cap (Figs. 1A and 1B). We then use the corresponding Mars Orbiter Laser Altimeter (MOLA) elevation profiles to correct the images for topography, yielding a record of image brightness versus vertical depth, a technique used in previous studies (Laskar et al., 2002; Milkovich and Head, 2005). The slope of the trough walls is $4.4^{\circ} \pm 0.6^{\circ}$, so the $300 \mathrm{~m}$ horizontal spacing of MOLA points is equivalent to $23 \pm 3$ vertical meters (mean \pm standard deviation). Although the High Resolution Imaging Science Experiment (HiRISE) offers superior resolution, we use MOC images because concurrent topographic profiles are available for a large number of sites. Preliminary studies of HiRISE images (Fishbaugh et al., 2008) suggest that MOC images resolve the finest-scale visible beds in the NPLD, but that HiRISE stereo topography will be useful in extending the present analysis.

Twelve prominent bedding contacts are identifiable in all six images (Fig. 1C). For a given pair of contacts, we calculate the mean and variance of the six measures of bed thickness. The slope of the relationship between thickness variance and mean thickness (Fig. 1D) provides a measure of the uncertainty, or "jitter," in the stratigraphic model (Huybers and Wunsch, 2004). Our jitter estimate of $0.25 \mathrm{~m}$ is a lower bound because it does not account for the possible presence of systematic variations in the time-depth relationship due to deformation, erosion, or variable accumulation rates, and because it is derived from images in close proximity to one another. Indeed, larger differences in stratigraphy have been observed over longer distances across the north polar cap (Fishbaugh and Hvidberg, 2006). Our jitter estimate does not account for topographic variations that affect the apparent thickness of beds thinner than the $23 \pm 3 \mathrm{~m}$ vertical spacing of the MOLA points, which are observed in stereo topography derived from a single pair of HiRISE images (Fishbaugh et al., 2008). However, the variance at these wavelengths is sufficiently small that the effect on our jitter estimate is negligible.

In addition to uncertainties in the stratigraphic model, the signal amplitude (image brightness) undoubtedly contains noise. The most significant noise sources are image artifacts and the indirect relationship between visible albedo and PLD composition. The visible albedo of the PLD recorded in the images is not determined by composition alone, but is also affected by illumination, surface roughness, and $\mathrm{CO}_{2}$ frost (Tanaka, 2005; Fishbaugh and Hvidberg, 2006; Herkenhoff et al., 2007). In some cases, even 


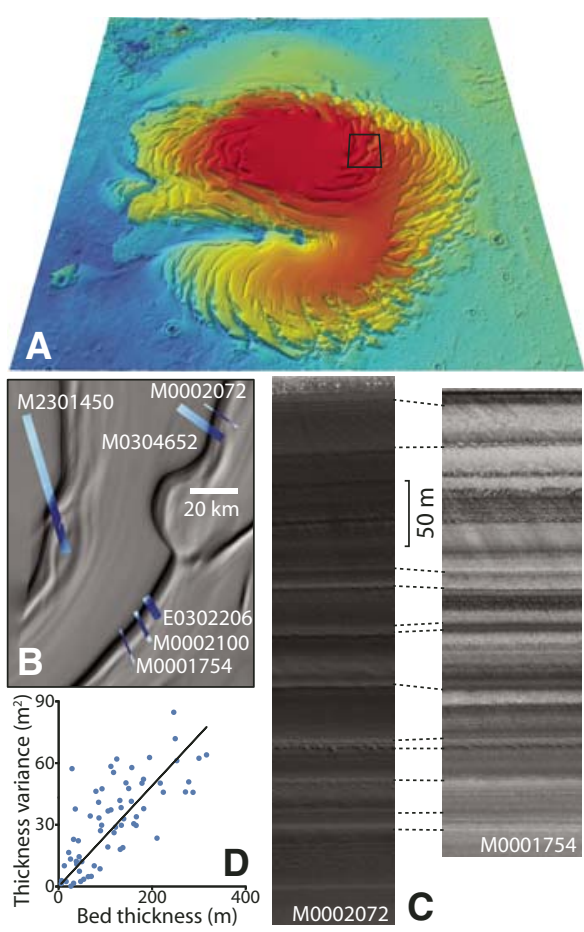

Figure 1. A: Perspective view of the north polar cap with elevation as color and black rectangle marking the boundaries of $B$. Scene in A is $1400 \mathrm{~km}$ across. B: A detailed view centered at $86.5^{\circ} \mathrm{N}, 89.7^{\circ} \mathrm{E}$, showing the locations of the six images used to measure jitter. C: The 12 bedding contacts visible in all six images, with two images shown as an example. D: Plot of bed thickness variance versus mean thickness. The slope of the regression line is the jitter.

the sign of the relationship is inconsistent, as illustrated by some beds that are lighter than their surroundings in some images, but darker in others (Fig. 1C). There are also indications that the NPLD may contain erosionally resistant beds that are not apparent in images (Fishbaugh et al., 2008). The full extent of the observational noise is uncertain, but we explore how reasonable estimates combine with jitter to degrade a pristine orbital signal.

The orbital signal we choose is the average daily insolation at $85^{\circ} \mathrm{N}$ on the summer solstice, which we calculate from the orbital solution of Laskar et al. (2004) using methods described by Berger (1978). For a range of NPLD accumulation rates suggested in the literature (Laskar et al., 2002, and references therein), we sample this insolation time series over intervals and at resolutions corresponding to the depth range and resolution of the image-based reconstructions of NPLD stratigraphy. For example, a typical image spans 400 vertical meters with a resolution of $20 \mathrm{~cm}$. For an accumulation rate of $0.2 \mathrm{~mm} / \mathrm{yr}$, the corresponding insolation record ranges from $2 \mathrm{Ma}$ to the present with a resolution of $1000 \mathrm{yr}$.
We corrupt the insolation signal by jittering the depths and by adding noise. To add jitter, we construct a random walk, $X_{n+1}=X_{n}+\eta \sqrt{\delta \phi}$, where $\delta=0.2 \mathrm{~m}$ is the depth resolution, $\eta$ is zeromean Gaussian noise with unit variance, and $\phi$ is a jitter of $0.25 \mathrm{~m}$, and add $X$ to the depths in the insolation record. The resulting unevenly spaced record is resampled at an even spacing. We then add red noise - a random signal in which longer wavelengths have larger amplitudes-to the insolation signal itself. The noise is constructed as a first-order autoregressive process with lag-1 autocorrelation and variance equal to those of the insolation record. We then compute power spectra for both the unperturbed and the jittered, noisy records using a standard periodogram approach. (For convenience we use the term "power spectra" with the understanding that the periodograms are estimates of the true power spectra.)

Figure 2 shows the results of this exercise for accumulation rates of $0.02 \mathrm{~mm} / \mathrm{yr}, 0.2 \mathrm{~mm} / \mathrm{yr}$, and $2 \mathrm{~mm} / \mathrm{yr}$, which span the range of estimated rates. Peaks corresponding to the $51 \mathrm{kyr}$ precession and $120 \mathrm{kyr}$ obliquity cycles in summer solstice insolation intensity are clearly visible in the unperturbed spectra (Figs. 2A-2C), except that the record with an accumulation rate of $2 \mathrm{~mm} / \mathrm{yr}$ is too short to resolve the obliquity cycle. Addition of our lower-bound jitter estimate and signal noise (Figs. 2D-2F) dramatically reduces the concentration of energy in high-frequency orbital bands and obscures the peaks in all three spectra, so that the overall spectral shape more closely resembles a noisy power-law trend than a background continuum punctuated by peaks. Thus, even in this relatively mild case- - with simple signals, a conservative estimate of jitter, and moderate noise-it becomes difficult to detect an orbital signature.

We judge that an unambiguous detection requires at least two significant concentrations of spectral energy with a ratio of periods corresponding to that of orbital variations. A ratio of periods is required because there are no independent constraints on the age-depth relationship within the PLD, such that the wavelengths at which periodic signals should occur are unknown. Given that some fraction of a spectrum is, by definition, expected to exceed a given confidence level by chance, a set of spectral peaks with a diagnostic ratio of periods would also be evidence that the peaks are truly significant. The obliquity cycle is not resolved by the record with the fast accumulation rate (Fig. 2A), nor is the precession signal distinguishable from the background after jitter and noise are added (Fig. 2D). For the slow accumulation rate (Fig. 2C), the obliquity and precession frequencies are only a few times smaller than the Nyquist frequency of the image-based stratigraphy, and are therefore especially susceptible to smearing by jitter (see Huybers and Wunsch, 2004). As a result, the

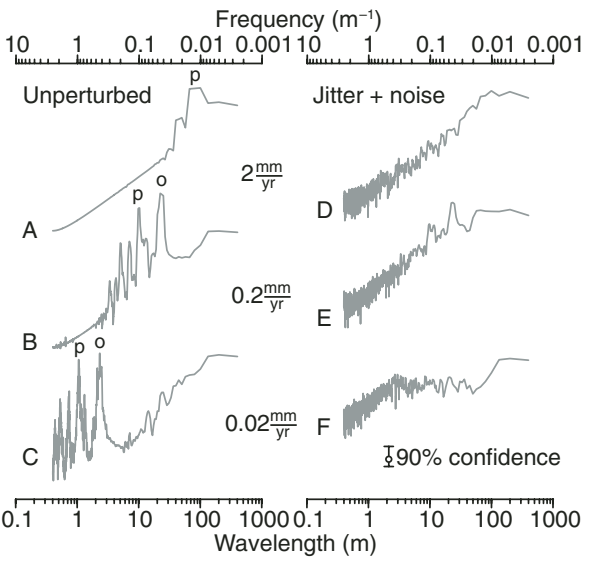

Figure 2. Power spectra for insolation time series with lengths and resolutions corresponding to a typical MOC image, for a range of polar layered deposits (PLD) accumulation rates. Spectra are plotted against wavelength rather than period to simulate the spectra that would be estimated from the PLD stratigraphy. The first-order trend was removed from each record prior to computing the FFT. Spectra for each accumulation rate have been multiplied by an arbitrary constant to create a vertical offset, and smoothed with a three-point moving average. Peaks corresponding to the precession (p) and obliquity (o) cycles are labeled in A-C. In D-F, jitter and red noise have been introduced in the signal. The vertical bar shows the $90 \%$ confidence interval for the power estimate at each wavelength with six degrees of freedom.

precession peak cannot be distinguished from the background, and the obliquity peak becomes a broad rise in power with marginal significance (Fig. 2F). Only for the intermediate accumulation rate are significant concentrations of spectral energy apparent at both the obliquity and precession bands in the noisy spectrum (Fig. 2E).

This analysis of synthetic stratigraphic records indicates that while jitter, noise, and short record duration do not categorically preclude the detection of an orbital signal, they do make it difficult. We now turn toward applying these insights to the interpretation of the actual NPLD stratigraphy.

\section{SPECTRAL ANALYSIS OF PLD STRATIGRAPHY}

To test for the presence of periodic signals within the PLD, we search for spectral peaks that deviate significantly from the expected shape of a noise spectrum. Using the method described in the previous section, we construct profiles of image brightness versus depth for 24 images from around the north polar cap, and compute the power spectrum for each profile. In all the spectra, power increases with wavelength until it levels off at the longest wavelengths (Fig. 3), consistent with a lag-1 autoregressive process- 


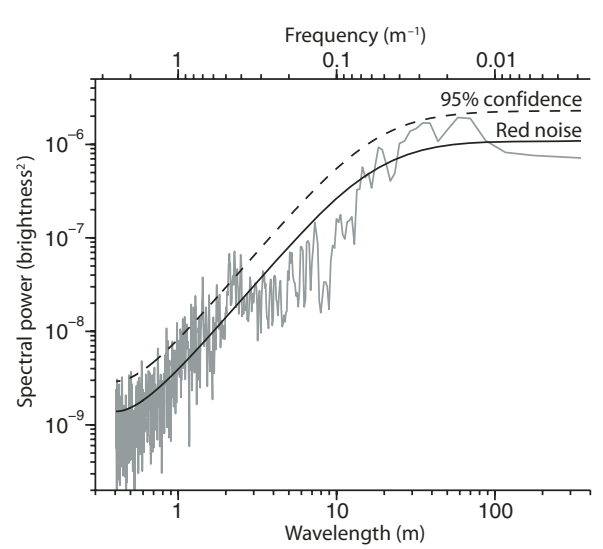

Figure 3. Periodogram for a vertical profile through MOC image M0001754, along with the theoretical spectrum and $95 \%$ confidence level for a red noise process (Gilman et al., 1963; Percival and Walden, 1993) with variance and lag-1 autocorrelation equal to those measured from the image profile.

perhaps the simplest red noise distribution. There are, however, two deviations relative to a simple red noise spectrum: First, at intermediate wavelengths of roughly 5-10 m, the image spectra have systematically lower power than the red noise spectra; and second, most of the image spectra contain broad peaks centered at wavelengths of a few meters that exceed the 95\% confidence level for red noise (Fig. 3). The consistency among images from various parts of the north polar cap suggests that these are real features of the NPLD.

As observed in the synthetic signal analysis, narrow-band processes with short wavelengths are especially susceptible to jitter and tend to be smeared across a broad range of frequencies. To explore the stratigraphic features associated with the high-frequency variability, we calculate wavelet transforms of the image profiles using methods described by Torrence and Compo (1998). Of the 24 wavelet spectra, 19 indicate intermittent bedding sequences with variability concentrated at bed thicknesses of a few meters. The spectrum in Figure 4 is typical of those 19. We obtain a measure of this characteristic thickness in each of the 19 images by identifying the peak in the depth-averaged wavelet spectrum that exceeds the $95 \%$ confidence level for red noise by the greatest fractional amount. The mean of these thicknesses is $1.6 \pm 0.1 \mathrm{~m}$. The string of peaks corresponding to these beds drifts toward shorter wavelengths with increasing depth in many images (Fig. 4), suggesting that the strata are compacted with depth or that the thickness of accumulating layers has increased through time.

\section{DISCUSSION AND CONCLUSIONS}

A fundamental limitation to interpreting the PLD climate record is the lack of an absolute chronology. The stratigraphy reconstructed

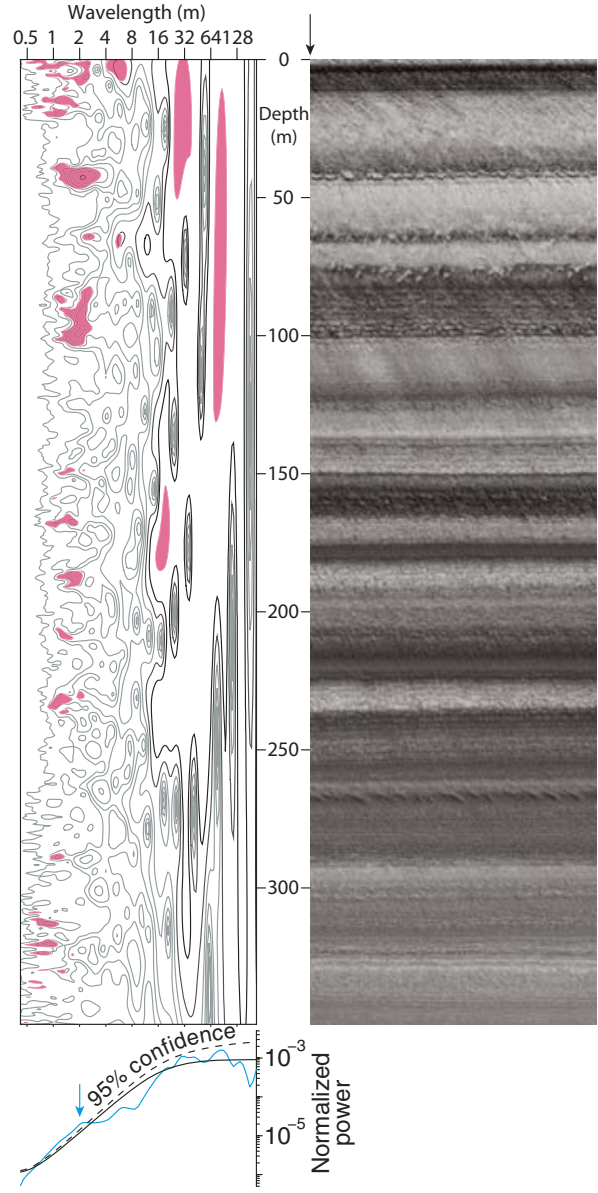

Figure 4. Wavelet power spectrum for a vertical profile through MOC image M0001754, with contours of spectral power (normalized to unit variance) at logarithmic intervals from $2^{-4}$ to $2^{4}$. Arrow at top of image shows the location of the vertical profile, which corresponds to the MOLA ground track. Image has been corrected for topography such that depth increases linearly along the vertical axis. Pink highlights show portions of the spectrum that exceed the $95 \%$ confidence level for red noise. Peaks more energetic than expected for a simple red noise background occur at wavelengths of 1-2 m where uniform bedding is visible in the image. The global wavelet spectrum below shows the average over the depth profile (blue line), with the corresponding red noise spectrum (black line), 95\% confidence level (dashed line), and arrow indicating the largest exceedance of the noise spectrum.

and analyzed here fails to resolve this problem because no clear connection can be made with a forcing function with a known time scale. The variance in the image-based stratigraphy is largely consistent with a stochastic formation process - one that is either random, or sufficiently nonlinear that it cannot be detected using conventional spectral methods.

Nonetheless, the $1.6 \mathrm{~m}$ bedding could be an orbitally forced signal that is distorted by noise, stratigraphic jitter, and nonlinearities in the rela- tionship between insolation and stratigraphy. There is some resemblance between the structures of the image spectra (such as the example in Fig. 3) and that of the jittered and noisy $20 \mathrm{Myr}$ insolation spectrum (Fig. 2F). If the $1.6 \mathrm{~m}$ bedding formed in response to obliquity or precession forcing, it would imply accumulation rates of $0.014-0.032 \mathrm{~mm} / \mathrm{yr}$ and a total formation time of roughly 30-70 Myr for the upper portion of the NPLD (unit $\mathrm{ABb}_{1}$ of Tanaka et al., 2008), which has a typical thickness of $1 \mathrm{~km}$. This formation time is within the upper bound of $\sim 1$ Gyr imposed by the underlying geologic units (Tanaka et al., 2008).

Alternatively, the $1.6 \mathrm{~m}$ bedding may have been formed by processes that are not directly related to orbital forcing, such as interannual variability in dust deposition (e.g., Haberle, 1986). We note that phenomena in Earth's climate, such as the El Niño Southern Oscillation and Dansgaard-Oeschger events, tend to concentrate variance at a particular time scale without being directly forced by orbital variations, and can also be intermittent. If the $1.6 \mathrm{~m}$ beds represent time intervals much shorter than orbital periods, it is possible that the NPLD formed within the last few million years, a scenario that has been proposed on the basis of climate modeling (Levrard et al., 2007) and radar observations of the large-scale stratigraphy of the NPLD (Phillips et al., 2008).

A robust estimate of absolute age would require multiple periodic bedding signals with a ratio of wavelengths that is diagnostic of known orbital periods. If the $1.6 \mathrm{~m}$ bedding is an obliquity signal, for example, we might expect to find a precession peak at $0.7 \mathrm{~m}$. At present, however, the $1.6 \mathrm{~m}$ bedding is the only significant deviation from a simple red noise continuum that is systematically observed throughout the NPLD. This result differs from that of Milkovich and Head (2005), who report that the NPLD contain a dominant wavelength of roughly $30 \mathrm{~m}$, but do not identify any short-wavelength signals. They implicitly assume that the spectral background is white noise-in which average power is the same at all wavelengths - and that the wavelength band with the highest power (tens of meters) represents the largest exceedance of this background, and therefore the most periodic signal. But a white noise background is inconsistent with the PLD spectra, which invariably have more power at longer wavelengths (Fig. 3). Our analysis also differs from that of Laskar et al. (2002), who derive an age estimate for the NPLD by tuning the brightness-depth record derived from one image to match the insolation record. Tuning the age model of even a wholly stochastic record to an assumed orbital forcing will generally create spurious orbital-like variability (Huybers and Wunsch, 2004), and in the spirit of Hays et al. (1976), we suggest that the presence of an orbital 
signal must first be demonstrated in a record that has not been orbitally tuned.

Three possible explanations for why the PLD spectra fail to show a distinct orbital signal are: (1) the orbital influence is negligible, (2) our image-based reconstruction of the stratigraphy contains biases, or (3) the relationship between orbital variations and the stratigraphy is nonlinear and is not detectable with the linear spectral techniques we employ. Given the large amplitude of orbitally induced variations in insolation (Ward, 1973; Laskar et al., 2004) and the predicted sensitivity of polar ice to these variations (Toon et al., 1980), we expect orbital variations to influence the stratigraphy, and thus expect that explanation 1 is insufficient. Given the demonstrated effects of our estimated jitter and of noise, explanation 2 alone could be sufficient. The $1.6 \mathrm{~m}$ bedding has more energy than expected for a simple red noise process, but the origin and implications of this variability are unclear.

We expect that explanation 3 is also sufficient to explain our inability to detect a distinct orbital signature. Studies using coupled models of the regolith and atmosphere (e.g., Toon et al., 1980), atmospheric general circulation models (e.g., Basu et al., 2006), and long-term observations of atmospheric dust dynamics (Zurek and Martin, 1993) suggest that Mars' climate system, while simpler than Earth's, can display significant nonlinearities. Cutts and Lewis (1982) and Levrard et al. (2007) show how relatively simple assumptions about PLD formation mechanisms can produce stratigraphy that is discontinuous and only weakly periodic. Moreover, they point out that one would expect a close correspondence between stratigraphy and orbitally forced insolation only in special circumstances, such as a scenario in which ice is deposited at a constant rate while the concentration of a small amount of dust is modulated by insolation-but this scenario seems improbable since ice is a volatile compound.

Given the expected difficulty of recovering even a simple orbital signal from the currently available record of the PLD stratigraphy, with its inherent jitter and noise, it is unsurprising that no clear orbital signature is evident. The meterscale bedding observed in the NPLD is suggestive and merits further exploration, but at present the stratigraphy appears more consistent with a stochastic signal than a set of superimposed periodic signals. Obtaining better and longer stratigraphic records, improved topography, and remotely sensed composition would permit a more stringent test of whether the PLD reflect variations in the orbital configuration of Mars.

\section{ACKNOWLEDGMENTS}

Perron was supported by a Reginald A. Daly Postdoctoral Fellowship. Huybers was supported by National Science Foundation award OCE-0645936.
We thank Carl Wunsch for his comments on an earlier version of the paper, and the three anonymous reviewers for their suggestions.

\section{REFERENCES CITED}

Basu, S., Wilson, J., Richardson, M., and Ingersoll, A., 2006, Simulation of spontaneous and variable global dust storms with the GFDL Mars GCM: Journal of Geophysical Research, v. 111, E09004, doi: 10.1029/2005JE002660.

Berger, A.L., 1978, Long-term variations of daily insolation and Quaternary climatic changes: Journal of the Atmospheric Sciences, v. 35, p. 2362-2367, doi: 10.1175/1520-0469(1978) 035<2362:LTVODI $>2.0 . C O ; 2$.

Cutts, J.A., and Lewis, B.H., 1982, Models of climate cycles recorded in Martian polar layered deposits: Icarus, v. 50, p. 216-244, doi: 10.1016/0019-1035(82)90124-5.

Cutts, J.A., Blasius, K.R., Briggs, G.A., Carr, M.H., Greeley, R., and Masursky, H., 1976, North polar region of mars: Imaging results from Viking 2: Science, v. 194, p. 1329-1337, doi: 10.1126/science. 194.4271.1329.

Fishbaugh, K.E., and Hvidberg, C.S., 2006, Martian north polar layered deposits stratigraphy: Implications for accumulation rates and flow: Journal of Geophysical Research, v. 111, E06012, doi: 10.1029/2005JE002571.

Fishbaugh, K.E., Byrne, S., Herkenhoff, K.E., Russell, P.S., Kirk, R.L., and McEwen, A.S., 2008, Characterizing and defining layers in the Martian north polar deposits using HiRISE: Implications for climate change: 39th Lunar and Planetary Science Conference, 10-14 March 2008, League City, Texas: Houston, Texas, Lunar and Planetary Institute, p. 1781.

Gilman, D.L., Fuglister, F.J., and Mitchell, J.M., Jr., 1963, On the power spectrum of "red noise": Journal of the Atmospheric Sciences, v. 20, p. 182-184, doi: 10.1175/1520-0469(1963) $020<0182$ :OTPSON $>2.0 . \mathrm{CO} ; 2$.

Haberle, R.M., 1986, Interannual variability of global dust storms on Mars: Science, v. 234, p. 459-461, doi: 10.1126/science.234.4775.459.

Hays, J.D., Imbrie, J., and Shackleton, N.J., 1976, Variations in the Earth's orbit: Pacemaker of the ice ages: Science, v. 194, p. 1121-1132, doi: 10.1126/science.194.4270.1121.

Herkenhoff, K.E., Byrne, S., Russell, P.S., Fishbaugh, K.E., and McEwen, A.S., 2007, Meterscale morphology of the north polar region of Mars: Science, v. 317, p. 1711-1715, doi: 10.1126/science. 1143544 .

Howard, A.D., Cutts, J.A., and Blasius, K.R., 1982, Stratigraphic relationships within Martian polar cap deposits: Icarus, v. 50, p. 161-215, doi: 10.1016/0019-1035(82)90123-3.

Huybers, P., and Wunsch, C., 2004, A depth-derived Pleistocene age model: Uncertainty estimates, sedimentation variability, and nonlinear climate change: Paleoceanography, v. 19, PA1028, doi: 10.1029/2002PA000857

Kominz, M.A., and Pisias, N.G., 1979, Pleistocene climate: Deterministic or stochastic?: Science, v. 204, p. 171-173, doi: 10.1126/science.204. 4389.171.

Laskar, J., Levrard, B., and Mustard, J.F., 2002, Orbital forcing of the Martian polar layered deposits: Nature, v. 419 , p. $375-377$, doi: 10.1038/nature01066.

Laskar, J., Correia, A.C.M., Gastineau, M., Joutel, F., Levrard, B., and Robutel, P., 2004, Long term evolution and chaotic diffusion of the insolation quantities of Mars: Icarus, v. 170, p. 343-364, doi: 10.1016/j.icarus.2004.04.005.

Levrard, B., Forget, F., Montmessin, F., and Laskar, J., 2007, Recent formation and evolution of northern Martian polar layered deposits as inferred from a Global Climate Model: Journal of Geophysical Research, v. 112, E06012, doi: 10.1029/2006JE002772.

Milkovich, S.M., and Head, J.W., 2005, North polar cap of Mars: Polar layered deposit characterization and identification of a fundamental climate signal: Journal of Geophysical Research, v. 110, E01005, doi: 10.1029/2004JE002349.

Murray, B.C., Soderblom, L.A., Cutts, J.A., Sharp, R.P., Milton, D.J., and Leighton, R.B., 1972, Geological framework of the south polar region of Mars: Icarus, v. 17, p. 328-345, doi: 10.1016/0019-1035(72)90004-8.

Percival, D.B., and Walden, A.T., 1993, Spectral analysis for physical applications: Multitaper and conventional univariate techniques: Cambridge, UK, Cambridge University Press, $583 \mathrm{p}$.

Phillips, R.J., and 26 others, 2008, North polar deposits: Stratigraphy, age, and geodynamical response: Science, v. 320, p. 1182-1185, doi: 10.1126/science. 1157546 .

Pollack, J.B., Colburn, D.S., Flasar, F.M., Kahn, R., Carlston, C.E., and Pidek, D., 1979, Properties and effects of dust particles suspended in the Martian atmosphere: Journal of Geophysical Research, v. 84, p. 2929-2945, doi: 10.1029/ JB084iB06p02929.

Tanaka, K.L., 2005, Geology and insolation-driven climatic history of Amazonian north polar materials on Mars: Nature, v. 437, p. 991-994, doi: 10.1038/nature04065.

Tanaka, K.L., Rodriguez, J.A.P., Skinner, J.A., Bourke, M.C., Fortezzo, C.M., Herkenhoff, K.E., Kolb, E.J., and Okubo, C.H., 2008, North polar region of Mars: Advances in stratigraphy, structure, and erosional modification: Icarus, v. 196, p. 318-358, doi: 10.1016/ j.icarus.2008.01.021.

Thomas, P., Squyres, S., Herkenhoff, K., Howard, A., and Murray, B., 1992, Polar deposits of Mars, in Kieffer, H.H., et al., eds., Mars: Tucson, University of Arizona Press, p. 767-795.

Toon, O.B., Pollack, J.B., Ward, W., Burns, J.A., and Bilski, K., 1980, The astronomical theory of climatic change on Mars: Icarus, v. 44, p. $552-$ 607, doi: 10.1016/0019-1035(80)90130-X.

Torrence, C., and Compo, G.P., 1998, A practical guide to wavelet analysis: Bulletin of the American Meteorological Society, v. 79, p. 61-78, doi: 10.1175/1520-0477(1998)079 $<0061$ :APGTWA>2.0.CO;2.

Ward, W.R., 1973, Large-scale variations in the obliquity of Mars: Science, v. 181, p. 260-262, doi: 10.1126/science.181.4096.260.

Wunsch, C., 2004, Quantitative estimate of the Milankovitch-forced contribution to observed Quaternary climate change: Quaternary Science Reviews, v. 23, p. 1001-1012, doi: 10.1016/j.quascirev.2004.02.014.

Zurek, R., and Martin, L., 1993, Interannual variability of planet-encircling dust storms on Mars: Journal of Geophysical Research, v. 98, p. 3247-3259, doi: 10.1029/92JE02936.

Manuscript received 1 June 2008

Revised manuscript received 9 October 2008

Manuscript accepted 13 October 2008

Printed in USA 